\title{
Analytical modelling of the expansion of a solid obstacle interacting with a radiative shock
}

Th. Michel ${ }^{1}$, E. Falize ${ }^{2,3}$, B. Albertazzi ${ }^{1}$, G. Rigon ${ }^{1}$, Y. Sakawa ${ }^{4}$, T. Sano ${ }^{4}$, H. Shimogawara ${ }^{4}$, R. Kumar ${ }^{4}$, T. Morita ${ }^{5}$, C. Michaut ${ }^{6}$, A. Casner $^{7}$, P. Barroso ${ }^{8}$, P. Mabey ${ }^{1}$, Y. Kuramitsu' ${ }^{9}$, S. Laffite ${ }^{2}$, L. Van Box Som ${ }^{2,10,3}$, G. Gregori ${ }^{11}$, R. Kodama ${ }^{9}$, N. Ozaki ${ }^{9}$, P. Tzeferacos ${ }^{12}$, D. Lamb ${ }^{12}$, and M. Koenig ${ }^{1,9}$

${ }^{1}$ LULI - CNRS, École Polytechnique, CEA : Université Paris-Saclay; UPMC Univ Paris 06 : Sorbonne Universités - F-91128 Palaiseau Cedex, France

${ }^{2}$ CEA, DAM, DIF, F-91297 Arpajon, France

${ }^{3}$ CEA Saclay, DSM/Irfu/Service d'Astrophysique, F-91191 Gif-sur-Yvette, France

${ }^{4}$ Institute of Laser Engineering, Osaka University, Suita, Osaka 565-0871, Japan

${ }^{5}$ Faculty of Engineering Sciences, Kyushu University, 6-1 Kasuga-Koen, Kasuga, Fukuoka 816-8580, Japan

${ }^{6}$ LUTH, Observatoire de Paris, PSL Research University, CNRS, Université Paris Diderot, Sorbonne Paris Cité, 92190 Meudon, France

${ }^{7}$ Université de Bordeaux-CNRS-CEA, CELIA, UMR 5107, F-33405 Talence, France

${ }^{8}$ GEPI, Observatoire de Paris, PSL Research University, CNRS, Université Paris Diderot, Sorbonne Paris Cité, 75014 Paris, France

${ }^{9}$ Graduate School of Engineering, Osaka University, Suita, Osaka 565-0871, Japan

${ }^{10}$ LERMA, Observatoire de Paris, PSL Research University, CNRS, Sorbonne Universités, UPMC Univ. Paris 06, F-75005 Paris, France

${ }^{11}$ Clarendon Laboratory, University of Oxford, Parks Road, Oxford OX1 3PU, UK

${ }^{12}$ Flash Center for Computational Science, University of Chicago, IL 60637, USA

(Received 14 November 2017; revised 19 March 2018; accepted 29 March 2018)

\begin{abstract}
In this paper, we present a model characterizing the interaction of a radiative shock (RS) with a solid material, as described in a recent paper (Koenig et al., Phys. Plasmas, 24, 082707 (2017)), the new model is then related to recent experiments performed on the GEKKO XII laser facility. The RS generated in a xenon gas cell propagates towards a solid obstacle that is ablated by radiation coming from the shock front and the radiative precursor, mimicking processes occurring in astrophysical phenomena. The model presented here calculates the dynamics of the obstacle expansion, which depends on several parameters, notably the geometry and the temperature of the shock. All parameters required for the model have been obtained from experiments. Good agreement between experimental data and the model is found when spherical geometry is taken into account. As a consequence, this model is a useful and easy tool to infer parameters from experimental data (such as the shock temperature), and also to design future experiments.
\end{abstract}

Keywords: high energy density physics; laser-plasmas interaction; modelling; plasmas astrophysics; plasma physics; radiative hydrodynamics; radiative shock

\section{Introduction}

Radiative shocks (RSs) are ubiquitous in astrophysics. They can be found in many phenomena, such as cataclysmic variables $^{[1]}$, supernovae or young stellar objects ${ }^{[2]}$. When a shock propagates above a threshold velocity, its radiation becomes so intense that it modifies its structure and the upstream electron density of the medium. This threshold

Correspondence to: Th. Michel, LULI, Ecole Polytechnique, Route de Saclay, 91128 Palaiseau, France. Email: thibault.michel@ polytechnique.edu velocity depends on the propagation medium properties, especially its equation of state and opacity. RSs are complex to model or to simulate due to, for example, the multiple length scales (from microscopic mean free paths to hydrodynamics lengths) involved. Moreover, direct astronomical observations do not give data on the RS dynamics. Today, RS can be generated in the laboratory with high-energy laser facilities, providing a better understanding of their properties.

In addition, the high radiative flux emitted by the shock can mimic radiation from O-stars near molecular clouds. 
Hence, the molecular cloud is ionized and photo-evaporates, creating ablation fronts that are difficult to model ${ }^{[3-6]}$. As a consequence, we introduce an obstacle a few $\mathrm{mm}$ away from the RS front, in order to experimentally study its ablation by a high radiative flux.

To ensure that the generated shocks are radiative, one must calculate two dimensionless numbers for the given experimental conditions: the Boltzmann number $\mathrm{Bo}$, which is the ratio between the thermal flux and the radiative flux, and the Mihalas number $R$, which is the ratio between the thermal energy and the radiative energy ${ }^{[7]}$. Thermal flux, radiative flux, as well as thermal energy and radiative energy are defined in Ref. [7] or [8]. Often in the astrophysical case, $B o \ll 1$, and hence high shock velocities are necessary in the laboratory in order to compare to the astrophysical case. Having $B o<1$ implies that the shock velocity is above a given threshold. This threshold velocity depends on the mass density and the atomic number of the propagation medium ${ }^{[9]}$. Indeed, in order to have the RS velocity comfortably such that $B o<1$, the propagation medium needs to be a high-Z low density material such as a gas. When $B o<1$, radiation emitted by the shock is absorbed in the upstream medium, the temperature rises and the material is ionized, inducing an increase in the electron density. This region, relative to a thick-thin shock, called the radiative precursor, has a smooth temperature gradient when it is optically thin (a temperature plateau when optically thick), and is thus very different from the pure hydrodynamical case. However, for the mass density, the usual sharp discontinuity at the shock front remains as the radiative energy is too low to modify its structure. Indeed, only when $R<1$ can a continuous mass density around the shock front occurs ${ }^{[9]}$. More generally, such a regime would change the whole hydrodynamics in the system.

Studies at the LULI2000 facility have observed the radiative precursor ${ }^{[10,11]}$, as well as fundamental parameters of RS generated in the laboratory (temperature, radial expansion, electron density, shock and precursor velocity). Other effects have been studied by the University of Michigan group on the OMEGA laser facility (Ref. [12] and references therein). In particular, a radiative collapse of a shock has been observed, as well as the strong interaction of radiation with the tube walls containing the gas where the RS propagated ${ }^{[13,14]}$.

The experiments performed in this paper were on GEKKO XII (Osaka, Japan), allowing the creation of a strong RS (velocities up to $160 \mathrm{~km} / \mathrm{s}$ ). An obstacle was introduced a few mm away from the initial shock front to observe the interaction between the RS and the obstacle. The main purpose of this design is to make sure that the RS parameters (especially its emission) are well understood and controlled, by studying the obstacle as a probe. It is also a way to characterize matter ablation due to the high radiative flux absorbed by the solid obstacle, increasing its temperature and inducing its expansion in the propagating medium. First, the experimental setup is described. In the second part, an analytical model to simulate the expansion of the obstacle as a function of time is proposed. This model is then used to compare numerical simulations and experimental data, showing that the model is an efficient way to describe the processes involved. Finally, the model is put forward as a quick and easy tool to design upcoming experiments, in complement to numerical simulations.

\section{Experimental setup}

We recently performed an experiment on the GEKKO XII HIPER laser facility in Osaka, Japan ${ }^{[15]}$, generating a strong RS showing its interaction with a $20 \mu \mathrm{m}$ aluminium foil. In a newer experiment, a quartz microballoon (provided by General Atomics) was utilized as the obstacle, being closer to the astrophysical case than a planar obstacle, in order to analyse its shape evolution. To generate the strong shock, the 9 beams of GEKKO XII are used with an energy of $1.2 \mathrm{~kJ}$, a pulse duration of $500 \mathrm{ps}$ at the wavelength of $351 \mathrm{~nm}$ and a $400 \mu \mathrm{m}$ diameter Gaussian focal spot, giving an intensity of $2 \times 10^{15} \mathrm{~W} \cdot \mathrm{cm}^{-2}$.

The laser beams irradiate a multilayer target $(\mathrm{CH} / \mathrm{Au} / \mathrm{Ti})$ where the gold layer acts as a hard X-ray shield to prevent preheating. The solid target operates as the strong shock generator that will break out and then propagate into a gas contained in a cell. In front of the shock, one may find a radiative precursor depending on the propagating medium (see above). The cell is filled with a high- $\mathrm{Z}$ low density gas (in our case, xenon at $31 \mathrm{mbar}$ ) to enhance the radiative effects as mentioned above. The quartz microballoon, having a $1 \mathrm{~mm}$ diameter and $10 \mu \mathrm{m}$ wall thickness, is situated $2 \mathrm{~mm}$ away from the multilayer target, within the Xe cell.

The multilayer target was designed in order to block hard X-ray (above $1 \mathrm{keV}$ ) from corona plasma ${ }^{[15]}$. As a consequence, the obstacle is still cold (much below $1 \mathrm{eV}$ ) when the shock breaks out of the solid target into the gas avoiding unwanted effects on the obstacle.

In the experiment, a large array of visible diagnostics is implemented in order to study the RS, its propagation, the radiative precursor and the obstacle behaviour. Figure 1 shows the experimental setup and the associated diagnostics. Transverse shadowgraphy (and interferometry) measurements were taken using a probe beam (few $\mathrm{mJ}, 532 \mathrm{~nm}$ and $10 \mathrm{~ns}$ duration), coupled with a gated optical imager (GOI), with an exposure time of $120 \mathrm{ps,}$ as well as with streaked cameras. Plasma at a density over $10^{20} \mathrm{~cm}^{-3}$ appears opaque on the shadowgraphy diagnostic (Figure 2(a)). Emission of the RS is characterized by a self-emission diagnostic. These diagnostics (presented in Figure 1) made it possible to determine several fundamental RS variables: its velocity, its upstream density and its temperature ${ }^{[16]}$. 


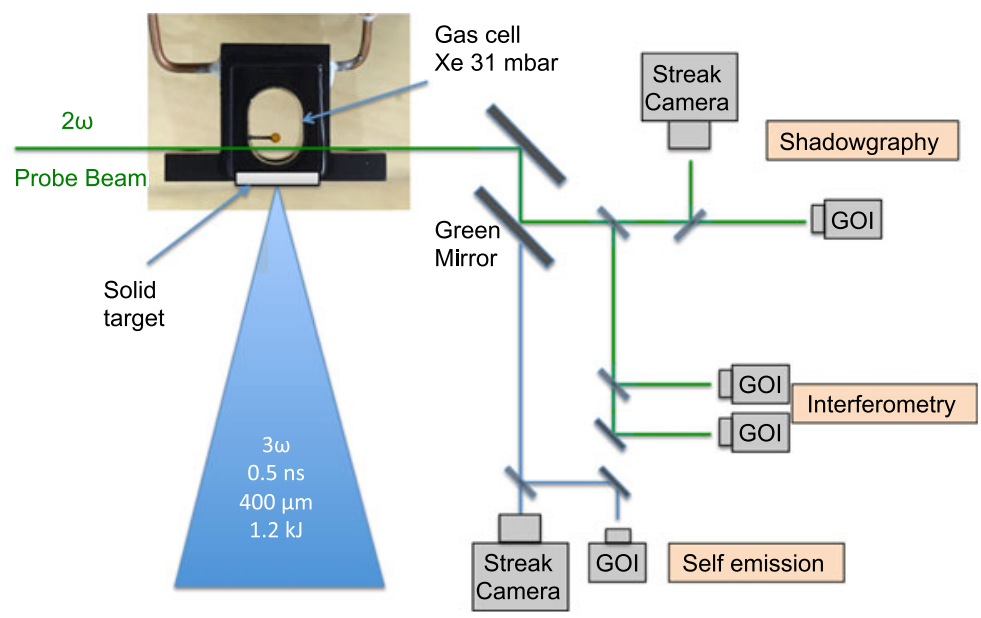

Figure 1. General setup of the experiment including all visible diagnostics.

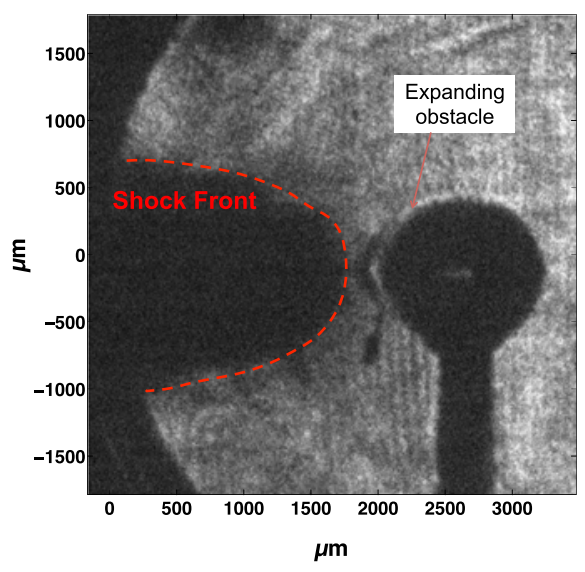

(a)

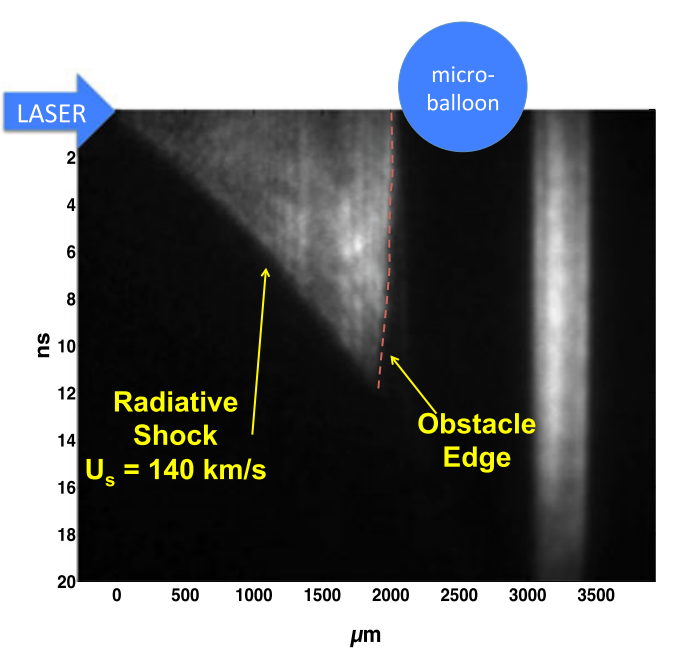

(b)

Figure 2. Shadowgraphy of the RS moving towards the obstacle. (a) A 2D snapshot obtained $10 \mathrm{~ns}$ after the drive beams; (b) streaked image that follows the position of the absorbing surface with time.

Figure 2(a) shows a 2D shadowgraphy snapshot obtained with a GOI. The RS propagates from left to right, towards a quartz microballoon used as the obstacle. The balloon no longer appears spherical, as it expands towards the RS due to radiation. To track the obstacle expansion versus time, the streaked shadowgraphy is used to follow the obstacle edge as a function of time (Figure 2(b)).

In this experiment, the obstacle's expanding overdense surface is the relevant parameter to determine and compare to the model. After presenting the general principles of the model, it is compared to the experimental data shown in Figure 2(b), by tracking the obstacle edge position. One has to note that the shadow in Figure 2(b) corresponds to electron density above $10^{20} \mathrm{~cm}^{-3}$.

\section{Model for the obstacle expansion}

\subsection{Principles of the modelling}

The aim of the model is to quantify the obstacle expansion, due to its ablation by the strong radiation coming from the shock front.

The distance between the shock and the obstacle is $D(t)=$ $D_{0}-u_{s} t$, where $D_{0}$ is the initial distance, $u_{s}$ the shock velocity and $t$ the time. We assume that $u_{s}$ is constant, a valid assumption as the shock launched in the gas is almost ballistic due to the low density of the medium.

The shock can be either planar or hemi-spherical, depending on the geometry, with a radius $R_{S}$ and a post-shock region that is fully opaque. We assume that the shock front radiates 


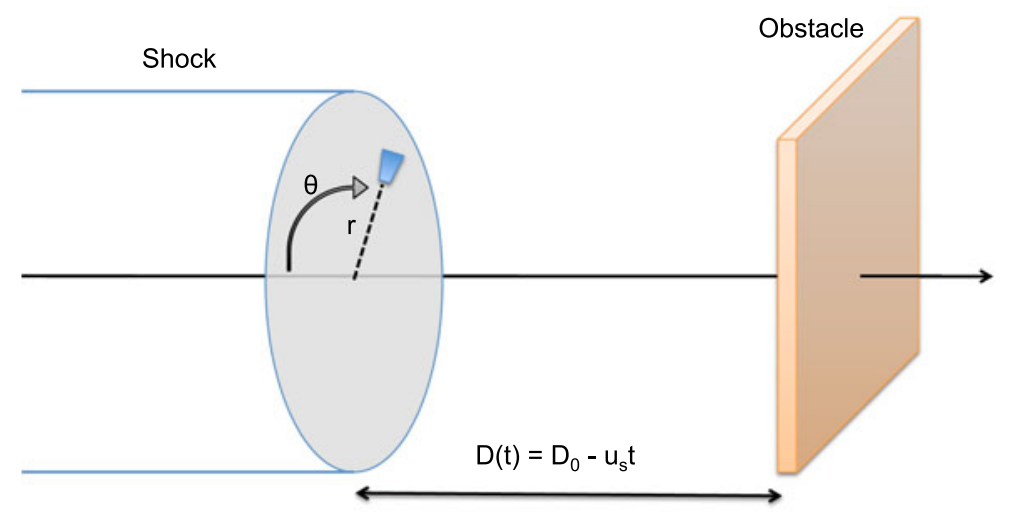

Figure 3. Schematic of the shock moving towards the obstacle.

as a black body, with a surface power $\phi_{e}=\sigma T_{s}^{4}$, where $T_{s}$ is the shock temperature and $\sigma=5.67 \times 10^{-8} \mathrm{~W} \cdot \mathrm{m}^{-2} \cdot \mathrm{K}^{-4}$ the Stefan-Boltzmann constant. This assumption is valid because the optical depth in the shocked region satisfies the equation $\tau \gg 1$, as the photons' mean free path is around $1 \mu \mathrm{m}$, much smaller than the hydrodynamic scale.

The obstacle can be either a $20 \mu \mathrm{m}$ thick aluminium foil $^{[15]}$ or a quartz microballoon. The expansion process is the following: the obstacle absorbs radiation coming from the shock, and its temperature rises as energy is deposited. The heated obstacle then expands towards the shock.

In the model presented here, we assume that the radiation energy is homogeneously absorbed by the obstacle at a distance (along the shock direction) $L_{\text {abs }}$. One notes that $L_{\mathrm{abs}}$ depends on the radiation wavelength. However, we assume here that the radiation is due to a single wavelength at an energy of $2.81 \times T_{S}[\mathrm{eV}]$, which is the energy corresponding to the maximum power emitted by a black body (Wien's law). This is for the ease of simplicity without changing the main results. At a given wavelength and for a given material, the typical absorption length $L_{\text {abs }}$, which depends on the obstacle temperature $T$ and mass density $\rho$, can be easily calculated. Cold opacities (as provided by The Center for X-Ray Optics X-ray interactions with matter calculator website (CXRO)) are used, as the obstacle is not heated by a significant amount. This gives the attenuation length of a radiation into a material at a given energy in $\mathrm{eV}$.

However, in the modelling, the obstacle opacity $\left(\rho L_{\text {abs }}\right)^{-1}$ is the only relevant parameter (see below), and has a low dependency on the temperature and density. In this paper, we consider $\rho L_{\text {abs }}$ to be constant during the obstacle expansion, as we assume a $1 \mathrm{D}$ expansion at the centre of the obstacle on the RS propagation axis. This is a valid assumption here as the expansion length is much smaller than the balloon diameter. If the obstacle expands on a length comparable to the obstacle diameter, then 2D effects implying a modification of $\rho L_{\text {abs }}$ must be taken into account. Moreover, we do not take into account the obstacle ionization effects, which can modify the quartz opacity. We assume $L_{\mathrm{abs}}=100 \mathrm{~nm}$ and $\rho=2.65 \mathrm{~g} \cdot \mathrm{cm}^{-3}$ in our experimental context. The obstacle is considered as a perfect gas, regarding its temperature, and it follows an isothermal expansion. This assumption is useful to link the absorbed energy and the temperature, the expansion velocity to the sound speed, leading to an expansion velocity varying as a function of $T$.

Indeed, we have

$$
v_{\exp }(t)=\sqrt{\frac{\gamma k_{B} T(t)}{\mu m_{H}}}
$$

with $k_{B}$ the Boltzmann constant, $\mu m_{H}$ the reduced mass and $\gamma$ the adiabatic index. The temperature evolution is given by

$$
\mathrm{d} E=\frac{3 R \rho L_{\mathrm{abs}} S}{2 M} \cdot \mathrm{d} T
$$

with $\mathrm{d} E$ the incoming energy in the obstacle, $\mathrm{d} T$ its temperature variation, $R$ the ideal gas constant, $M$ the molar mass and $S$ the expanding surface. Equation (2) shows that calculating the incident energy on the obstacle as a function of time leads to the determination both of its temperature and of its expansion velocity (Equation (1)).

\subsection{Planar shock}

Here, we consider a planar shock propagating towards the obstacle (Figure 3). Every surface element of coordinates $(r, \theta)$ emits a power of

$$
\mathrm{d} \phi_{e}=\sigma T_{s}^{4} \times r \mathrm{~d} r \mathrm{~d} \theta
$$

This radiation is isotropic, and radiates everywhere. Here, we focus on the radiation absorbed at the centre of the obstacle, on the RS axis. Indeed, this is where radiation is the most important so it will expand faster than anywhere else. It is also where the RS is imaged onto the streak camera slit.

The surface $S$ at the centre of the obstacle absorbs a ratio $\Omega / 2 \pi$ of all the power $\mathrm{d} \phi_{e}$ emitted by a surface element of 


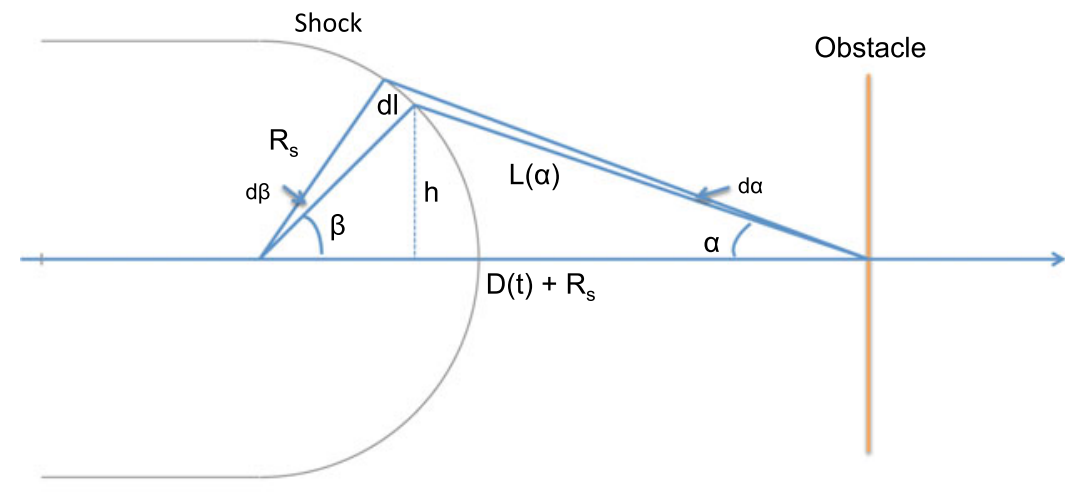

Figure 4. Spherical shock moving towards the obstacle.

coordinates $(r, \theta)$ (see above). Here, $\Omega=S /\left(D(t)^{2}+r^{2}\right)$ is the solid angle of the absorbing surface seen from the emitting surface element. After integrating on the planar RS surface, we get

$$
\phi_{r}(t)=\frac{\sigma T_{s}^{4}}{2} \ln \left[1+\left(\frac{R_{S}}{D(t)}\right)^{2}\right] .
$$

Equation (4) gives the energy absorbed by the obstacle during a given time. By using Equation (2) and integrating it between 0 and $t$, one can show that

$$
\begin{aligned}
T(t)= & T_{0}+\frac{\sigma T_{s}^{4} \mu m_{H}}{3 \gamma k_{B} \rho L_{\mathrm{abs}}}\left\{\frac{D_{0}}{u_{s}} \ln \left[1+\left(\frac{R_{s}}{D_{0}}\right)^{2}\right]\right. \\
& -\left(\frac{D_{0}}{u_{s}}-t\right) \ln \left[1+\left(\frac{R_{s}}{D_{0}-u_{s} t}\right)^{2}\right] \\
& \left.+2 \frac{R_{s}}{u_{s}} \arctan \left[\frac{R_{s} u_{s} t}{R_{s}^{2}+D_{0}\left(D_{0}-u_{s} t\right)}\right]\right\} .
\end{aligned}
$$

Combining this with Equation (1), we get

$$
\begin{aligned}
v_{\mathrm{exp}}^{2}(t)= & \frac{\sigma T_{s}^{4}}{3 \rho L_{\mathrm{abs}}}\left\{\frac{D_{0}}{u_{s}} \ln \left[1+\left(\frac{R_{s}}{D_{0}}\right)^{2}\right]\right. \\
& -\left(\frac{D_{0}}{u_{s}}-t\right) \ln \left[1+\left(\frac{R_{s}}{D_{0}-u_{s} t}\right)^{2}\right] \\
& \left.+2 \frac{R_{s}}{u_{s}} \arctan \left[\frac{R_{s} u_{s} t}{R_{s}^{2}+D_{0}\left(D_{0}-u_{s} t\right)}\right]\right\} .
\end{aligned}
$$

We arrive at an analytical formula giving the expansion velocity as a function of time and a function of several variables. Most of these variables are known experimentally with accuracy. Indeed, $D_{0}, u_{s}$, and $R_{s}$ are determined through 2D or streaked shadowgraphy. Thanks to selfemission diagnostics, a relative shock temperature may be determined, but due to a lack of calibration, its absolute value is not attainable. Experimentally, it can only be determined that $T_{s} \in[20 \mathrm{eV} ; 40 \mathrm{eV}]$. Thus, we assume that $T_{s}=$ $30 \mathrm{eV}$, which is also justified by $2 \mathrm{D}$ radiative hydrodynamics FLASH simulations (see below).

One can note that the expansion velocity grows rapidly with the shock temperature $\left(T_{s}^{4}\right)$. Moreover, the obstacle expands faster if its surface mass ( $\left.\rho L_{\mathrm{abs}}\right)$ is smaller. Finally, the expansion velocity increases with time, as we assume here a constant energy flux radiated by the shock front.

This model, in planar geometry, can provide accurate expansion velocity of the obstacle when the experiment or astrophysical situations can be approximated to this particular geometry. However, this is rarely the case, so we have developed a dedicated model for a spherical shock, being more complex, but more suited to experiments or astrophysics.

\subsection{Spherical shock}

The motivation for this section is to compare the spherical shock to the planar shock referring to the obstacle expansion velocity as the main parameter. Indeed, if the expansion velocity is similar for both cases, then the planar shock analytical formula can be used to compare to data, and to fit experimental results, as the model is much simpler than the spherical case.

The model in the spherical case is more complex to establish, but is more consistent with experiment (cf. Figure 2(a)).

First, given the cylindrical symmetry, one needs to calculate the total emission from the ring on the RS characterized by the coordinates $[\alpha ; \alpha+\mathrm{d} \alpha]$ (all notation are explained on Figure 4). The surface of this ring is $2 \pi h \mathrm{~d} l$, where $h=L \sin \alpha$.

Moreover, we get $\mathrm{d} l$ from pure geometrical arguments. Indeed,

$$
\mathrm{d} l=R_{s} \mathrm{~d} \beta,
$$

and

$$
L \sin \alpha=R_{S} \sin \beta .
$$




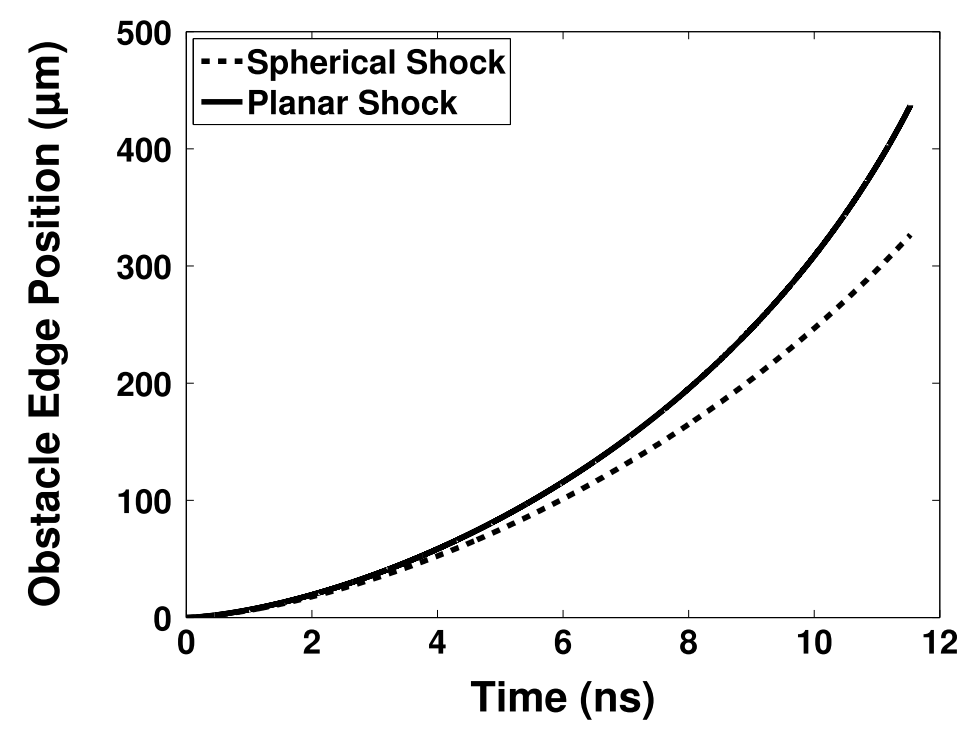

Figure 5. Comparison between a spherical and a planar shock. The shock velocity is $140 \mathrm{~km} / \mathrm{s}$ at $30 \mathrm{eV}, R_{S}=500 \mu \mathrm{m}$.

In addition

$$
L \sin (\alpha+\mathrm{d} \alpha)=R_{s} \cos (\beta+\mathrm{d} \beta) .
$$

Assuming that $\cos (\mathrm{d} \alpha)=\cos (\mathrm{d} \beta)=1, \sin (\mathrm{d} \alpha)=\mathrm{d} \alpha$, $\sin (\mathrm{d} \beta)=\mathrm{d} \beta$ and using Equations (7) and (8) yields

$$
\mathrm{d} l=L \frac{\cos \alpha}{\sqrt{1-\left(\frac{L}{R_{s}}\right)^{2} \sin ^{2} \alpha}} \mathrm{d} \alpha .
$$

Finally, the total emitted power from the ring is

$$
\mathrm{d} \phi_{e}=\sigma T_{s}^{4} \times 2 \pi L^{2} \frac{\cos \alpha \sin \alpha}{\sqrt{1-\left(\frac{L}{R_{s}}\right)^{2} \sin ^{2} \alpha}} \mathrm{d} \alpha .
$$

Thus, only a ratio $\Omega(\alpha) / 2 \pi$ is absorbed by the surface $S$ at the centre of the obstacle compared to the total emission from the ring, where $\Omega=S / L(\alpha)^{2}$ is the solid angle of the absorbing surface seen from the emitting surface element, similar to the planar case.

One can now write the flux density power $\phi_{r}$ received by the obstacle:

$$
\phi_{r}(t)=\sigma T_{s}^{4} \int_{0}^{\alpha_{\max }(t)} \frac{\cos \alpha \sin \alpha}{\sqrt{1-\left(\frac{L(\alpha, t)}{R_{s}}\right)^{2} \sin ^{2} \alpha}} \mathrm{d} \alpha,
$$

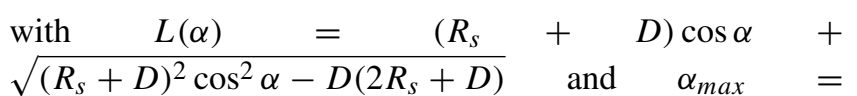
$\arcsin \left(\frac{R_{s}}{R_{s}+D}\right)$.

Now that one has the flux density power received by the obstacle, Equations (2) and (1) can be used to get the obstacle expansion velocity, just like in the planar case:

$$
v_{\exp }(t)=\sqrt{\frac{2}{3 \rho L_{\mathrm{abs}}} \int_{0}^{t} \phi_{r}\left(t^{\prime}\right) \mathrm{d} t^{\prime}}
$$

Our analytical model provides the obstacle expansion velocity as a function of time, with respect to several parameters, for both planar and spherical cases. It is now possible to compare these two geometries, plotting the obstacle edge position, as this parameter can be easily determined in the experiments (see Figure 2(b)).

In Figure 5, we compare the spatial expansion of the obstacle for an RS velocity of $140 \mathrm{~km} / \mathrm{s}$ having a temperature of $30 \mathrm{eV}$ and a shock front radius of $500 \mu \mathrm{m}$ interacting with an aluminium foil situated at $2 \mathrm{~mm}$ at $t=0$.

First, we observe that the two models show a similar behaviour, with a total expansion around $400 \mu \mathrm{m}$ after $12 \mathrm{~ns}$. However, in the planar case, it expands $100 \mu \mathrm{m}$ more compared to the spherical case, an observation that is compatible with the conservation of energy in the total system.

Thus, the model predicts an expansion velocity of the order of $30 \mathrm{~km} / \mathrm{s}$ compatible with our experimental results (see Figure 2(b) showing a final velocity of around $35 \mathrm{~km} / \mathrm{s}$ in the last $2 \mathrm{~ns}$ ) and with previous results ${ }^{[15]}$.

However, as mentioned in the Introduction, when the shock is highly radiative (in our experiments, using xenon gas at 31 mbar), the electron density in the upstream region increases due to a radiative flux emitted by the shock front much higher than the thermal one. To be consistent with the physics situation, this process also needs to be taken into account in the calculation of all radiation absorbed by the obstacle leading to its expansion. 


\subsection{Radiative precursor in the model}

Depending on the xenon density, part of the radiation emitted by the shock is absorbed by the gas, and cannot reach the obstacle at all unless the radiation mean free path is large enough. Therefore, the radiative precursor can be highly heated and emits some further thermal radiation. According to Ref. [17], the radiative density flux at the abscissa $z_{2}$ as a function of the density flux at $z_{1}$ can be written as

$$
\begin{aligned}
I\left(z_{2}\right)= & I\left(z_{1}\right) e^{-\left(z_{2}-z_{1}\right) / L_{\text {prec }}} \\
& +L_{\text {prec }}^{-1} \int_{z_{1}}^{z_{2}} B(z) e^{-\left(z-z_{1}\right) / L_{\text {prec }}} \mathrm{d} z
\end{aligned}
$$

where $L_{\text {prec }}$ is the medium absorption length (here, linked to the precursor length and experimentally equal to $600 \mu \mathrm{m}$ ) and $B(z)$ is the emission at $z$ which is, in the precursor, a fraction of the Planck function for black body emission. To take the precursor into account, Equation (14) needs to be plugged into Equation (13) by modifying the flux irradiating the obstacle. Instead of taking into account the geometrical dilution only, Equation (14) is used with $z_{1}$ the emission surface abscissa and $z_{2}$ the absorption surface abscissa. We assume a temperature profile following $T(z)=$ $T_{S}\left(1-\frac{z}{L_{\text {prec }}}\right)$ as in an optically thin medium, corresponding to our experimental case. One can note that for higher xenon pressure (few hundreds of mbar), this assumption cannot be made and the model is not valid anymore. For this case, we would need to assume a temperature profile in the precursor following $T(z)=T_{s} \times \tanh \left(z / L_{\text {prec }}\right)$, but the method remains the same. Without going into full details, this effect is part of the model and is taken into account for the results below. The precursor significantly affects the results, as the obstacle interacts less with the RS at first (as the precursor absorbs a non-negligible part of the power emitted by the RS), but at the end is heated by both the RS and the precursor. Finally, the obstacle expansion velocity as a function of time is more convex when the radiative precursor is taken into account.

\section{Results}

In this section, we discuss the obstacle expansion either from experimental data, from simulations or from the model presented above, in the spherical case and with a radiative precursor.

\subsection{Model validation}

In order to validate our model, we make a detailed comparison between the experimental data, our model and hydrodynamics simulations. For this last case, we use two different radiation-hydrodynamics codes to be compared to the model described above: MULTI, which is a 1D Lagrangian code ${ }^{[18]}$, and FLASH, a 2D/3D AMR code, solving the radiation equations with the diffusion approximation ${ }^{[19]}$. In order to compare experimental data and simulation results for a given shot, the code is constrained to reproduce the measured RS velocity. As a consequence, for all comparisons done in this paper, the shock velocity will remain $u_{s}=140 \mathrm{~km} / \mathrm{s}$. For both codes, we use the laser-matter interaction module to reproduce the experimental conditions. The energy groups in this multigroup simulation are also well refined near the shock temperature for the gas, near the pusher K-alpha edge. The non-LTE is also taken into account for coronal plasma.

Moreover, experimental data such as the shock diameter, the radiative precursor length, and the distance between the target and the obstacle will be the input parameters in our model, and are very well known from transverse diagnostics (Figures 2(a) and 2(b)). Regarding the shock temperature (which is a very important parameter to infer the obstacle expansion), we were able to determine it in the range [ $20 \mathrm{eV} ; 40 \mathrm{eV}$ ], as an absolute precise calibration was not possible as mentioned previously.

Figure 6 shows the obstacle expansion as a function of time related to one single shot, from experimental data (Figure 2), simulations and our model.

First, we can clearly see that all methods used to evaluate the obstacle expansion show the same behaviour regarding the obstacle expansion. Second, we observe that this expansion velocity increases with time leading to a final value of several hundreds of microns.

Regarding the simulations, the expansion inferred from the 1D code MULTI is higher than the one calculated from the model as well as the one measured experimentally. Indeed, as expected in a $1 \mathrm{D}$ code, temperature of the shock is overestimated, radiation losses not being properly taken into account $^{[20]}$.

In the $2 \mathrm{D}$ case, FLASH seems to under-estimate the expansion. It can be explained by a poor accuracy in the opacity table for xenon, a low number of groups in the multigroup approach around $100 \mathrm{eV}$, or a limited radiation module of FLASH (diffusion limit) compared to analytical solution which is obtained for the complete radiative transfer equation.

Finally, the obstacle expansion given by our model seems to reproduce accurately the experimental data. The compatibility between the inferred expansion with our model and the experimental one shows that all physical processes occurring in the experiment are well described.

One has to note that most of the parameters are experimentally well known $\left(u_{s}, R_{s}, D_{0}\right.$, etc.), except the shock temperature $T_{s}$, which ranges between $20 \mathrm{eV}$ and $40 \mathrm{eV}$. As a consequence, in the following, we characterize how this variable affects our model regarding the obstacle expansion. 


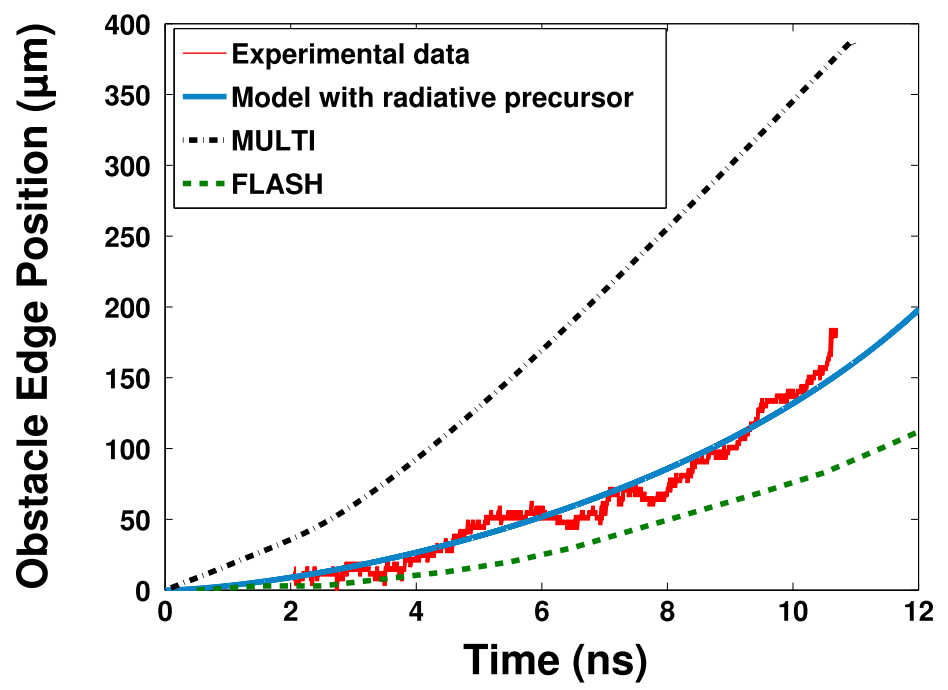

Figure 6. Comparison between model, experiment, and simulations. The model parameters, related to the experiment, are $T_{s}=30 \mathrm{eV}, u_{s}=140 \mathrm{~km} / \mathrm{s}$, a precursor length of $600 \mu \mathrm{m}$ and a shock diameter of $1 \mathrm{~mm}$.

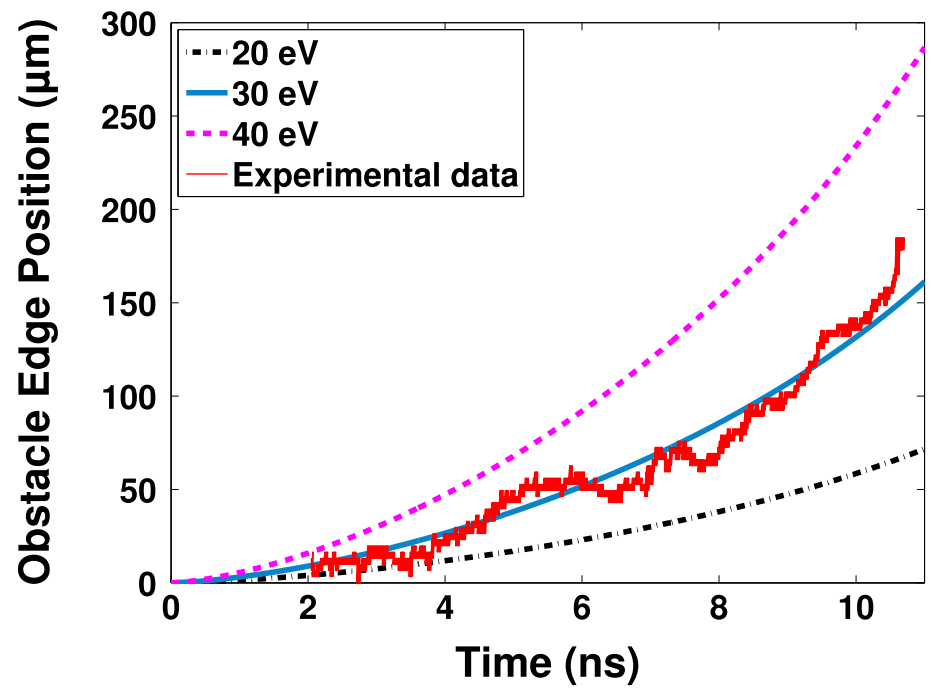

Figure 7. Same as Figure 6, with experimental data and model expansion with three temperatures $(20 \mathrm{eV}, 30 \mathrm{eV}$ and $40 \mathrm{eV})$.

\subsection{Effect of temperature}

To determine the influence of the shock temperature on the obstacle expansion, we plot on Figure 7 the calculated expansion for three different shock temperatures, still comparing it with the experimental data.

As expected, when the shock temperature is higher, the obstacle temperature increases as the radiative flux is enhanced; the consequence is that the expansion velocity, closely related to the sound velocity, increases as well. One can also observe in Figure 7 that the shock temperature must be close to $30 \mathrm{eV}$ to fit with the experimental data, the curve at $20 \mathrm{eV}$ or $40 \mathrm{eV}$ being too far from the results. As a consequence, thanks to our model, we can possibly discriminate the temperature in an RS experiment in the presence of an obstacle, confirming that the temperature is close to $30 \mathrm{eV}$ in our shot. One can also note that the temperature given by the FLASH simulation presented above is also close to $30 \mathrm{eV}$.

Another possibility to infer the exact temperature would be to use SESAME tables at a given xenon density and shock velocity ${ }^{[21]}$. However, the SESAME equation of state does not take into account the radiative losses and, as a consequence, over-estimates the shock temperature compared to the experimental one. Finally, an absolute and precise calibration of the self-emission diagnostic can be a way to determine the experimental temperature, technique that will be adopted in future experiments.

However, it must also be noted that this model is not only useful to determine some parameters (such as the shock temperature) after an experiment, but also to design upcoming experiments in order to optimize the obstacle 


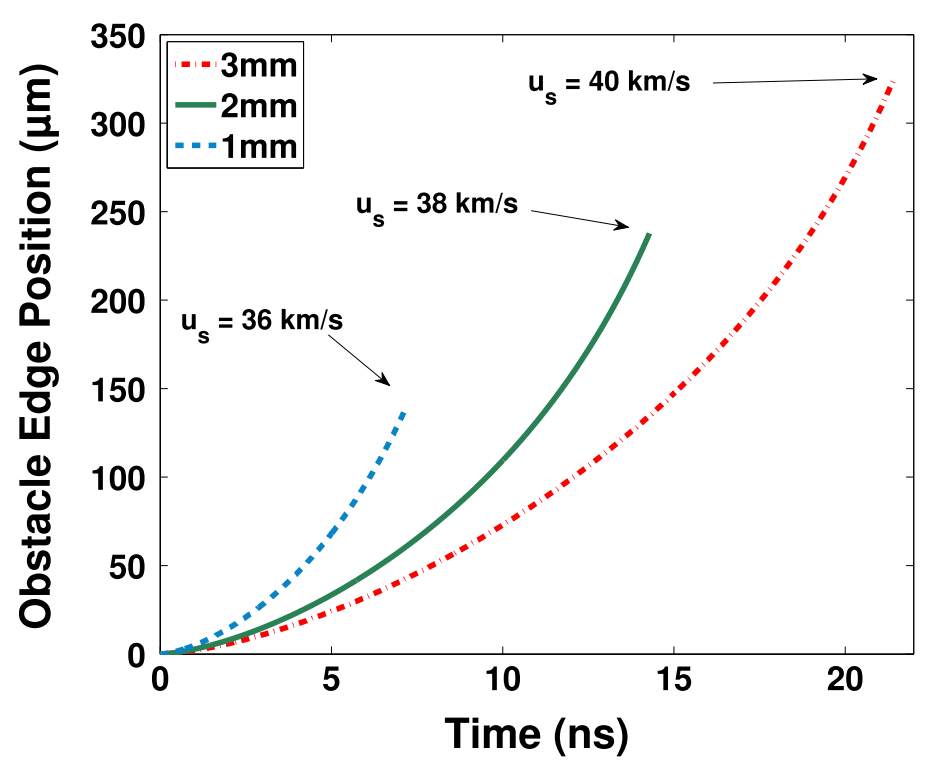

Figure 8. Same as Figure 7, with three different initial distances between the target and the obstacle $(1 \mathrm{~mm}, 2 \mathrm{~mm}, 3 \mathrm{~mm})$.

expansion. Unfortunately, the direct application of this particular model to a strictly astrophysical context is of limited use. Indeed, astrophysical RSs which propagate in the interstellar medium (made of hydrogen) are generally fully ionized, unlike laboratory experiments where the RS structure greatly depends on the xenon opacity (opacity mainly due to bound-bound and bound-free transition). These two behaviours are not in agreement with scaling laws application $^{[22]}$. This hypothesis is not valid in our model however.

\subsection{Future experiments}

Some upcoming RS experiments need to be prepared, especially at GEKKO XII and at LMJ. In all these experiments, the RS itself will be studied (for example, the presence of a radiative precursor, velocity, shape, ...), as well as the interaction with an obstacle.

In order to improve the experimental design, we intend to quantify the effect of the initial distance between the RS and the obstacle $D_{0}$.

Figure 8 shows the edge position calculated with our model for different initial distances between the RS and the obstacle. The other parameters are the same as exposed in Figure 6.

We can see that for longer distances, the obstacle expands farther: due to longer initial distances, the obstacle is irradiated for a longer time by the RS.

However, the expansion is faster at initial time for $D_{0}=$ $1 \mathrm{~mm}$ than for $D_{0}=3 \mathrm{~mm}$. Indeed, the radiative flux irradiating the obstacle is higher, inducing a higher temperature. After a while, for a long initial distance, the RS keeps heating the obstacle, so its temperature still increases as the RS gets closer to the obstacle. As a consequence, the final velocity becomes similar to the $1 \mathrm{~mm}$ case.

Indeed, we do note that the final velocity is almost the same for the three different cases (from $36 \mathrm{~km} / \mathrm{s}$ for $D_{0}=$ $1 \mathrm{~mm}$ to $40 \mathrm{~km} / \mathrm{s}$ for $D_{0}=3 \mathrm{~mm}$ ). In our case, the radiative precursor absorbs about $70 \%$ of the incoming flux emitted by the RS front. As a consequence, the obstacle is mostly heated when it interacts with the radiative precursor, i.e., when the RS is at a distance $L_{\text {prec }}$ from the obstacle.

In our RS experiments, we aim to observe a maximum expansion length, lasting for longer time, rather than the fastest expansion, as it is easier to determine its dynamics.

As a consequence, for future GEKKO experiments, we will put the obstacle at $3 \mathrm{~mm}$ from the target, because a larger expansion than the one observed in our shot shown in this paper will occur. This distance will allow the development of the RS on a longer distance, and thus the radiative effects will be easier to observe.

Thus, our model is a useful tool to aid in the preparation of upcoming experiments, being able to anticipate the obstacle expansion, and is therefore a quick and easy complementary tool together with 2D simulations.

\section{Conclusion}

In the context of RS experiments, we have developed an analytical model explaining an obstacle expansion situated at a given initial distance from a propagating RS. We have shown that this model, without any free parameters, predicts an expansion very close to the experimental data, showing the obstacle expansion process is well described. To achieve the consistency between experiment and modelling, the RS temperature is found to be around $30 \mathrm{eV}$, which is 
compatible with the temperature measured experimentally $\left(T_{s} \in[20 \mathrm{eV} ; 40 \mathrm{eV}]\right)$ and the one given by $2 \mathrm{D}$ radiative hydrodynamic simulations using the FLASH code. However, one has to note that the temperature here is seen as an initial condition and not as a predicted result. Finally, we have shown that our model can be easily used to plan experiments, as a quick complement to full 2D simulations.

This model can also be improved, by considering, for example, the obstacle shape (i.e, calculate the expansion all over the obstacle surface). A continuous spectrum for the shock emission and therefore the total energy absorbed by the obstacle for each wavelength emitted by the shock, can also be introduced.

In future experimental campaigns, we anticipate having access to the experimental temperature thanks to a precise calibration of self-emission diagnostics. As a consequence, an even more precise comparison between the model results and experimental data can be performed. Moreover, we will ensure that the designed initial distance, chosen via our model, will lead to a larger expansion. This detailed study of the obstacle expansion will help with better understanding of the RS itself, for example, to quantify the shock temperature as a function of the shock velocity by taking into account the radiative losses at the same time.

\section{Acknowledgements}

The authors would like to thank the ILE staff for their great support. The GEPI target fabrication has to be acknowledged, as well as General Atomics for providing the balloons. Part of this work was supported by the Scientific Council of the Observatoire de Paris and by COST (European COoperation in Science and Technology), action MP1208, with a Short-Term Scientific Mission.

\section{References}

1. C. Busschaert, E. Falize, C. Michaut, J. M. BonnetBidaud, and M. Mouchet, Astron. Astrophys. 579, A25 (2015).

2. S. Orlando, R. Bonito, C. Argiroffi, F. Reale, G. Peres, M. Miceli, T. Matsakos, C. Stehle, L. Ibgui, L. de Sa, J. P. Chieze, and T. Lanz, Astron. Astrophys. 559, A127 (2013).

3. L. Spitzer, Astrophys. J. 120, 1 (1954).

4. E. A. Frieman, Astrophys. J. 120, 18 (1954).
5. R. J. R. Williams, D. Ward-Thompson, and A. P. Whitworth, Mon. Not. R. Astron. Soc. 327, 788 (2001).

6. A. Mizuta, J. O. Kane, M. W. Pound, B. A. Remington, D. D. Ryutov, and H. Takabe, Astrophys. J. 621, 803 (2005).

7. C. Michaut, E. Falize, C. Cavet, S. Bouquet, M. Koenig, T. Vinci, A. Reighard, and R. P. Drake, Astrophys. Space Sci. 322, 77 (2009).

8. R. Drake, Astrophys. Space Sci. 298, 49 (2005).

9. S. Bouquet, R. Teyssier, and J. P. Chize, Astrophys. J. Suppl. Ser. 127, 245 (2000).

10. T. Vinci, M. Koenig, A. Benuzzi-Mounaix, C. Michaut, L. Boireau, S. Leygnac, S. Bouquet, O. Peyrusse, and D. Batani, Phys. Plasmas 13, 010702 (2006).

11. S. Bouquet, C. Sthl, M. Koenig, J.-P. Chize, A. BenuzziMounaix, D. Batani, S. Leygnac, X. Fleury, H. Merdji, C. Michaut, F. Thais, N. Grandjouan, T. Hall, E. Henry, V. Malka, and J.-P. J. Lafon, Phys. Rev. Lett. 92, 225001 (2004).

12. R. Drake, F. Doss, R. McClarren, M. Adams, N. Amato, D. Bingham, C. Chou, C. DiStefano, K. Fidkowski, B. Fryxell, T. I. Gombosi, M. J. Grosskopf, J. P. Holloway, B. van der Holst, C. M. Huntington, S. Karni, C. M. Krauland, C. C. Kuranz, E. Larsen, B. van Leer, B. Mallick, D. Marion, W. Martin, J. E. Morel, E. S. Myra, V. Nair, K. G. Powell, L. Rauchwerger, P. Roe, E. Rutter, I. V. Sokolov, Q. Stout, B. R. Torralva, G. Toth, K. Thornton, and A. J. Visco, High Energy Density Phys. 7, 130 (2011).

13. A. B. Reighard, R. P. Drake, K. K. Dannenberg, D. J. Kremer, M. Grosskopf, E. C. Harding, D. R. Leibrandt, S. G. Glendinning, T. S. Perry, B. A. Remington, J. Greenough, J. Knauer, T. Boehly, S. Bouquet, L. Boireau, M. Koenig, and T. Vinci, Phys. Plasmas 13, 082901 (2006).

14. F. W. Doss, H. F. Robey, R. P. Drake, and C. C. Kuranz, Phys. Plasmas 16, 112705 (2009).

15. M. Koenig, T. Michel, R. Yurchak, C. Michaut, B. Albertazzi, S. Laffite, E. Falize, L. Van Box Som, Y. Sakawa, T. Sano, Y. Hara, T. Morita, Y. Kuramitsu, P. Barroso, A. Pelka, G. Gregori, R. Kodama, N. Ozaki, D. Lamb, and P. Tzeferacos, Phys. Plasmas 24, 082707 (2017).

16. G. W. Collins, P. M. Celliers, L. B. Da Silva, R. Cauble, D. M. Gold, M. E. Foord, N. C. Holmes, B. A. Hammel, R. J. Wallace, and A. Ng, Phys. Rev. Lett. 87, 165504 (2001).

17. R. P. Drake, Phys. Plasmas 14, 043301 (2007).

18. R. Ramis, R. Schmalz, and J. Meyer-Ter-Vehn, Comput. Phys. Commun. 48, 475 (1988).

19. F. C. for Computational Science, FLASH User Guide (University of Chicago, 2015).

20. M. Koenig, T. Vinci, A. Benuzzi-Mounaix, N. Ozaki, A. Ravasio, M. R. le Glohaec, L. Boireau, C. Michaut, S. Bouquet, S. Atzeni, A. Schiavi, O. Peyrusse, and D. Batani, Phys. Plasmas 13, 056504 (2006).

21. SESAME: The LANL Equation of State Database, Report No. LA-UR-92- 3407 (University of Chicago, 1992).

22. É. Falize, C. Michaut, and S. Bouquet, Astrophys. J. 730, 96 (2011). 